\title{
Estructura y características de la agricultura coreana
}

DOI: $10.32870 /$ mycp.v11i31.301

Sujin $\operatorname{Lim}^{1}$

Resumen

El presente artículo analiza la evolución de la agricultura surcoreana en el periodo de posguerra haciendo énfasis en el impacto de la rápida industrialización del país en la escala de producción de las unidades productoras agrícolas y de la composición de la fuerza de trabajo. Se toma como punto de partida el impacto redistributivo de la reforma agraria y como hilo conductor, la relevancia del cultivo del arroz en la agricultura coreana.

Palabras clave: agricultura, desarrollo, reforma agraria, cultivo del arroz, escala de producción, fuerza de trabajo agrícola.

\section{Abstract}

This article analyses the evolution of South Korean agriculture during the post world period making emphasis on the impact of the rapid industrialization of the country on the scale of production of productive units and on the composition of the agricultural labor force. It takes as a point of departure the Agrarian Reform and its redistributive impact and as a center of the analysis the importance of rice in Korean agriculture.

Key words: Agriculture, development, agrarian reform, rice cultivation, production scale, agrarian workforce.

\section{Introducción}

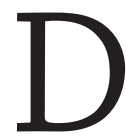
espués de la revolución industrial (finales del siglo XVIII), la actividad económica mundial se dirigió a la industrialización y a los servicios, en tanto que la agricultura disminuía; y aunque la actividad agrícola se relaciona directamente con la soberanía en materia de alimentos de un país, ésta se redujo en todas las naciones durante este periodo. Aún hoy en

1. Profesora investigadora de la Universidad de Colima. 
día, la actividad agrícola parece no tener tanta importancia en los países desarrollados.

Generalmente la importancia agrícola se mide en dos rangos: en el porcentaje de la población rural y en el PIB agrícola. En el caso de Corea, ${ }^{2} 7.4 \%$ de la población total es rural, mientras que en Japón, EE.UU., Gran Bretaña y Francia tienen $3.2 \%, 2.0 \%, 1.7 \%$ y $2.9 \%$, respectivamente. Con relación a la proporción de la producción agrícola entre producción total, en Corea, Japón, EE.UU., Gran Bretaña, y Francia se registraron para el año 2005, 4.5\%, 1.0\%, $2.0 \%, 1.0 \%$, y $3.0 \%$, respectivamente.

Hace cincuenta años, la agricultura era la principal actividad económica en Corea: más de 90\% de la Población Económicamente Activa (PEA) se dedicaba a esta actividad; asimismo, más de $50 \%$ del PIB nacional provenía de la agricultura. La agricultura funcionaba no sólo como suministro de alimentos, sino también como fuente de empleo y de presupuesto para el gobierno. Pero hoy en día, la agricultura en Corea está lejos de las funciones mencionadas anteriormente, más bien se le considera como un obstáculo para el desarrollo de ese país; problema que casi todos los países desarrollados presentan en esta época de globalización.

En el presente trabajo se analizan la estructura básica y las características de la agricultura en Corea, circunscribiéndonos al periodo que abarcan los últimos cincuenta años. La organización del artículo se presenta de la siguiente forma:

Primero se analiza la estructura o distribución de la propiedad, y con base en ello se muestran las características de la repartición de la tierra, enfocando la situación a la pequeña escala en el tamaño de la propiedad.

En segundo lugar, se hace un análisis de la estructura de la producción agrícola y se obtienen sus características; concretamente nos enfocamos en la importancia del arroz entre todos los productos, conociendo las particularidades que se originan de esta situación.

Por último, se examina la estructura social de la agricultura de Corea, especialmente se estudia el perfil demográfico y se obtiene la característica social. En concreto, se analiza la disminución de la población rural y el problema de su envejecimiento.

2. Nos referimos al caso de Corea del Sur. 
Estructura y característica de la propiedad agrícola en Corea

Generalmente, uno de los factores más importantes que caracteriza la estructura agrícola es la propiedad de la tierra. En el caso de EE.UU., el tamaño promedio es de 190 hectáreas (ha) y en Canadá llegan hasta 246, mientras que en Australia el promedio de la propiedad llega hasta 4000 ha. En el caso de Corea, el promedio de la propiedad queda en 1.3 ha. En una palabra, podemos decir que es demasiado pequeña, no sólo en $\mathrm{t}$ \{erminos relativos sino también absolutamente. Para explicar la situación de Corea, su pueden considerar dos perspectivas.

\section{Estructura física}

El territorio total de Corea es de $99,000 \mathrm{~km}^{2}$, en donde viven más de 48 millones habitantes. ${ }^{3}$ La densidad de la población por kilómetro cuadrado es de 485 personas, ocupando el tercer lugar del mundo, solo por debajo de Bangladesh (897 personas por kilómetro cuadrado) y Taiwán (617 personas por kilómetro cuadrado). Si consideramos la densidad poblacional en cada kilómetro cuadrado de EE.UU., Canadá, Australia, etc., la de Corea es muy alta. Además en el caso de Corea, el terreno adecuado para la agricultura ocupa sólo $18 \%$ del territorio total. Podemos decir que en menos de 20,000 kilómetros cuadrados de terreno se distribuyen más de 1.24 millones de unidades agrícolas (entendiéndose por unidad agrícola a una familia campesina). Por lo tanto, el tamaño de terreno que corresponde a cada unidad agrícola se ve reducido a una escala pequeña.

\section{Perspectiva histórica}

Al observar esta perspectiva, encontramos un ejemplo representativo: la reforma agrícola que se aplicó en el año de 1949 a 1950. Así, en el año 1945, cuando Corea se independizó de Japón, la estructura económica de este país estuvo encabezada por la agricultura, donde más de $90 \%$ de la PEA se dedicaba a esta actividad y más de $50 \%$ del PIB se obtenía de ella. Pero la situación de la agricultura fue muy débil por la polarización de la propiedad, puesto que en 1945 el terreno total cultivable era de 2'225,751 hectáreas, donde sólo 37\% era

3. Por ejemplo, el territorio total del estado Oaxaca es casi igual al de Corea. 
cultivado por su propio dueño y el otro $63 \%$ era trabajado por arrendatarios (familias campesinas sin tierras) que rentaban el terreno.

Por otra parte, el caso de la unidad agrícola familar fue aún más grave, ya que entre todas las unidades de producción agrícola sólo $13.7 \%$ cultivaban en su propio terreno, mientras que $34.6 \%$ tenían que rentar terreno extra para cultivar, esto debido a que sus propiedades eran demasiado pequeñas y tenían que buscar así la forma que les permitiera alcanzar la producción de consumo. En lo que respecta a las otras $49.8 \%$ unidades agrícolas familiares, éstas no tenían terreno alguno para cultivar, además el restante $2.6 \%$ de las familias que carecieron de unidad agrícola de familia tuvieron que emplearse como peones de labranza. En resumen, podemos afirmar que más de $50 \%$ de las unidades agrícolas de familias no contaban con tierra propia, por lo que tenían que rentarla para mantenerse.

En esta situación, el pago de arrendamiento era de 50\%; después de cosechar, los arrendatarios tenían que dar la mitad de sus ganancias a los propietarios. En ese tiempo, más de $80 \%$ de la población coreana residía en las localidades rurales, por lo que su economía permaneció en un círculo de pobreza del que no podía salir.

Frente a esta realidad, la reforma agraria aplicada en 1949 ofreció una gran oportunidad de carácter institucional a las familias campesinas arrendatarias, que formaban la mayor parte de las unidades de producción agrícola, al permitirles liberarse de la pobreza extrema. Tras la independencia (1945) el principal objetivo político del gobierno coreano fue el establecimiento de una sociedad más estable, pero en medio de una realidad desalentadora en donde la mayor parte de la población caía en el rango de arrendatario paupérrimo, el gobierno no pudo cumplir con su propósito. Para hacerlo posible, tuvo que intentar abatir la pobreza y aumentar la productividad agrícola mediante la privatización de la tierra. Finalmente, después de tres años, el gobierno estableció la Ley Agraria que estipulaba: "La tierra se distribuirá entre quienes realmente la trabajen". El método de distribución fue el de "confiscación y distribución gravosa", a diferencia de Corea del Norte que la distribuyó mediante la "confiscación y distribución gratuita". Con base en esta ley, el gobierno compró la tierra a los propietarios que tenían más de tres hectáreas y se las vendió a los arrendatarios. En ese tiempo 191,411 hectáreas de arrozales y 76,467 de hortalizas se distribuyeron gracias a la Ley de la Reforma Agraria, y 953,000 unidades agrícolas familiares recibieron el beneficio de la distribución (Kim, 2005). 
La reforma agraria permitió que la proporción de arrendatarios disminuyera de 63.4\% en 1945 a 40\% en 1949, hasta 11\% en 1960 (Chang, 2000). Debido a la Reforma Agraria, el fenómeno de concentración de la tierra desapareció y los campesinos pudieron tener su propia parcela; pero el tamaño del la propiedad tuvo que permanecer en un rango muy pequeño (tabla 1 ).

\section{Tabla 1}

Estructura agraria de la propiedad por tamaño de las unidades familiares en 1960

\begin{tabular}{ccccc}
\hline Menos de 0.5 ha & 0.5-1.0 ha & $1.0-2.0 h a$ & $2.0-3.0 h a$ & Más de 3.0 ha \\
\hline $42.9 \%$ & $30.1 \%$ & $20.7 \%$ & $6.0 \%$ & $0.3 \%$ \\
\hline
\end{tabular}

Fuente: Korea Rural Economic Institute (KREI, 2006).

La tabla 1 muestra la estructura de propiedad del terreno después de diez años de la reforma agraria. Como se puede observar en ella, más de $73 \%$ de las unidades de producción familiar agrícola permanecieron en el rango de tamaño de propiedad con menos de una hectárea, en tanto que el rango de más de tres hectáreas ocupa $0.3 \%$ del total. Con esto podemos decir que la mayor parte de la población agrícola tenía una propiedad menor a una hectárea.

Cabe señalar que esta característica de la pequeña propiedad permanece hasta hoy en día. Durante los últimos cincuenta años, la estructura económica en Corea ha cambiado dinámicamente a causa de la industrialización; sin embargo, en el caso de la agricultura, especialmente en la estructura de la propiedad, no se refleja esa variación (tabla 2).

\section{Tabla 2}

Distribución de la propiedad del terreno agrícola con relación al tamaño (unidad: ha)

\begin{tabular}{ccccccc}
\hline Año & $<0.5$ & $0.5-1.0$ & $1.0-1.5$ & $1.5-2.0$ & $2.0-3.0$ & $>3.0$ \\
\hline 1965 & 35.9 & 31.7 & 16.6 & 9.1 & 5.6 & 1.2 \\
1970 & 32.6 & 34.2 & 18.5 & 8.0 & 5.1 & 1.5 \\
1975 & 30.2 & 36.2 & 18.8 & 8.2 & 4.9 & 1.6 \\
1980 & 28.8 & 35.1 & 20.6 & 9.0 & 5.1 & 1.5 \\
1985 & 28.4 & 36.5 & 20.7 & 8.5 & 4.6 & 1.2 \\
1990 & 27.7 & 31.2 & 20.2 & 11.0 & 7.4 & 2.5
\end{tabular}


Sujin Lim

\begin{tabular}{ccccccc}
\hline Año & $<0.5$ & $0.5-1.0$ & $1.0-1.5$ & $1.5-2.0$ & $2.0-3.0$ & $>3.0$ \\
\hline 1995 & 29.3 & 29.3 & 18.0 & 10.3 & 8.4 & 4.8 \\
2000 & 32.3 & 27.7 & 16.0 & 9.6 & 8.3 & 6.2 \\
2004 & 36.5 & 26.4 & 14.5 & 8.4 & 7.5 & 6.7 \\
\hline
\end{tabular}

Fuente: KREI, 2006.

Como se muestra en la tabla 2, con relación a la propiedad de la tierra, la polarización aumentó con el tiempo, puesto que en las décadas de 1970, 1980 y 1990, el rango de menos de 0.5 hectáreas disminuyó, y desde la década de 1990 comenzó a incrementarse. Según datos de KREI, en el año 2004 más de $36 \%$ de unidades agrícolas familiares tenían menos de 0.5 ha de terreno. Durante 40 años los rangos de 0.5 a 1.0, 1.0 a 1.5, y 1.5 a 2.0 ha (escala o tamaño mediano) disminuyeron, no siendo estas diferencias significativas. Pero el rango de más de 3 ha (escala o tamaño grande) aumentó considerablemente durante los últimos 40 años. En el año 1965, 1.2\% del total de las unidades familiares agrícolas poseían más de 3 ha, pero en el año 2004 el porcentaje del rango de más de 3 ha subió hasta $6.7 \%$, podemos decir que se incrementó más de tres veces.

Aunque en el rango de más de 3 ha aumentó en los últimos 40 años, en la actualidad más de $63 \%$ de las unidades familiares agrícolas aún tienen menos de una hectárea, y esta situación puede ser un factor importante que caracterice a la agricultura de Corea. Lo anterior se aclara al comparar ésta con la situación de otros países (tabla 3).

Tabla 3

Comparación del promedio del tamaño de las unidades familiares por países seleccionados en el año 2005 (unidad: ha)

\begin{tabular}{llll}
\hline País & $\begin{array}{l}\text { Promedio de la } \\
\text { propiedad por unidad }\end{array}$ & País & $\begin{array}{l}\text { Promedio de la } \\
\text { propiedad por unidad }\end{array}$ \\
\hline EE.UU. & 190 & Alemania & 30 \\
Canadá & 246 & China & 2.1 \\
Australia & 4011 & Japón & 1.4 \\
Francia & 39 & Corea & 1.3 \\
\hline
\end{tabular}

Fuente: Kim, 2002. 
Como se ve en la tabla 3 , en comparación con países como EE.UU., Canadá y Australia, el tamaño de la propiedad de Corea es muy

Históricamente, el arroz ha sido considerado como el motor principal en la agricultura de Corea pequeño, y aunque los países asiáticos como China y Japón tienen un tamaño pequeño relativamente, Corea se ubica por debajo de estas naciones asiáticas, situación que caracteriza la estructura agrícola coreana.

Estructura y característica de la producción agrícola coreana: el arroz como pivote

Si resumimos la estructura y las características de la producción agrícola de Corea, podemos decir que el arroz es el principal producto agrícola. Cuando la agricultura ocupaba la mayor parte de la actividad económica, se decía que la economía coreana era igual que la economía del arroz. En Corea el arroz ha sido no sólo la comida básica, sino también el principal elemento constitutivo de su cultura e historia.

Históricamente, el arroz ha sido considerado como el motor principal en la agricultura de Corea. Su estructura y características pueden analizarse de acuerdo a la proporción del terreno en donde se cultiva, la proporción de la unidad familiar que lo cultiva entre todas las unidades familiares agrícolas, la proporción de las calorías que provienen de éste, entre todas las calorías de que se compone el consumo calórico total de la población, y su proporción en la producción total agrícola en Corea.

La proporción del terreno en donde se cultiva arroz entre todos los terrenos cultivables

En el año 2004 la tierra cultivable total era de 1'233,000 ha, de la cual, en 1’001,000 ha se cultivó arroz. Los arrozales ocupaban más de $80 \%$ del terreno cultivable. El fenómeno de concentración encabezado por el arroz se ha fortalecido con el tiempo (tabla 4).

Como se muestra en la tabla 4, en el año 1965 el terreno cultivable llegó hasta 2'950,000 ha, pero con la industrialización y la urbanización, el terreno cultivable disminuyó, y a finales de 2004 ocupó nada más 1'233,000 ha. La 
Tabla 4

Superficie destinada a la producción agrícola en Corea

(unidad: miles de ha \%)

\begin{tabular}{lrrrrrr}
\hline Año & Total & Arroz & Cebada & Soya & Papa & Otros granos \\
\hline 1965 & 2950 & 1228 & 933 & 362 & 213 & 214 \\
1970 & 2699 & 1203 & 834 & 358 & 181 & 123 \\
1975 & 2522 & 1218 & 761 & 324 & 146 & 73 \\
1980 & 1994 & 1233 & 360 & 244 & 92 & 53 \\
1985 & 1780 & 1237 & 242 & 196 & 65 & 40 \\
1990 & 1669 & 1244 & 160 & 188 & 40 & 37 \\
1995 & 1346 & 1056 & 90 & 132 & 40 & 28 \\
2000 & 1316 & 1072 & 68 & 107 & 44 & 25 \\
2004 & 1233 & 1001 & 63 & 100 & 40 & 27 \\
\hline Porcentaje & & & & & & \\
\hline 1965 & 100 & $42 \%$ & $32 \%$ & $12 \%$ & $7 \%$ & $7 \%$ \\
1970 & 100 & $45 \%$ & $31 \%$ & $13 \%$ & $7 \%$ & $5 \%$ \\
1975 & 100 & $48 \%$ & $30 \%$ & $13 \%$ & $6 \%$ & $3 \%$ \\
1980 & 100 & $62 \%$ & $18 \%$ & $12 \%$ & $5 \%$ & $3 \%$ \\
1985 & 100 & $69 \%$ & $14 \%$ & $11 \%$ & $4 \%$ & $2 \%$ \\
1990 & 100 & $75 \%$ & $10 \%$ & $11 \%$ & $2 \%$ & $2 \%$ \\
1995 & 100 & $78 \%$ & $7 \%$ & $10 \%$ & $3 \%$ & $2 \%$ \\
2000 & 100 & $81 \%$ & $5 \%$ & $8 \%$ & $3 \%$ & $2 \%$ \\
2004 & 100 & $81 \%$ & $5 \%$ & $8 \%$ & $3 \%$ & $2 \%$ \\
\hline
\end{tabular}

mFuente: elaboración propia con datos de Estadística Anual Agropecuaria 2005.

disminución del terreno cultivable afectó más fuertemente la producción de cebada, soya, papa y otros granos. En el caso de la cebada, disminuyó 15 veces durante los últimos 40 años. Entre el total de la superficie cultivable, la ocupación de la cebada disminuyó de $32 \%$ hasta $5 \%$ en el mismo periodo. Al igual que la cebada, la proporción de la soya, papa y otros granos disminuyó considerablemente en el mismo periodo.

Con relación a esta situación, la causa más importante fue la falta de mano de obra. El gran éxito de la industrialización en Corea demandaba intensivamente mano de obra, por lo que muchos campesinos dejaron sus actividades agrícolas para dirigirse a la zona urbana, abandonado de tal manera varios cultivos. 
Pero, al contrario de los casos anteriores, la proporción de arroz aumentó durante los últimos cuarenta años, y aunque la superficie de arrozales disminuyó, la proporción de este producto entre todos los cereales aumentó de 42\% hasta $81 \%$. En comparación con la velocidad de disminución del terreno de otros cereales, el arrozal no disminuyó tan rápido, situación que se muestra en el gráfico siguiente.

\section{Gráfico 1}

Superficie destinada a la producción agrícola en Corea

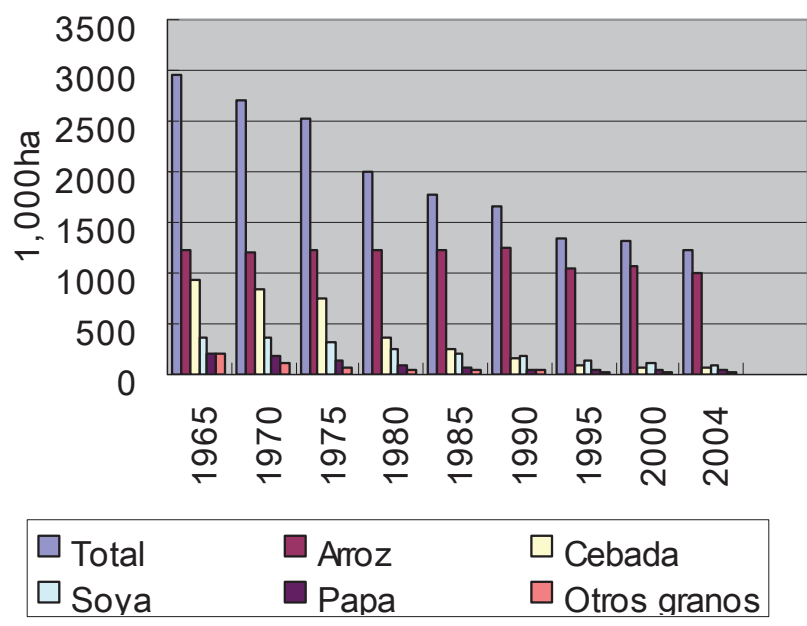

Fuente: Ministerio Agropecuario, 2005.

Como se ve en el gráfico 1 , mientras disminuía el terreno cultivable total de cebada, soya, papa y otros granos, la superficie del arroz no se redujo tanto. Hoy en día, el arroz se siembra en más de $80 \%$ del terreno cultivable total. Podemos decir que durante la industrialización y urbanización, la agricultura de Corea se redujo físicamente; sin embargo, el arroz se maneja casi igual que hace cuarenta años.

La proporción de la unidad familiar que se dedica a la producción del arroz, entre todas las unidades familiares agrícolas

Entre todas las unidades de familias agrícolas, la mayor parte se dedica al cultivo del arroz. Según datos del Ministerio Agrícola, en el año 2004, 74.7\% de las 
unidades familiares agrícolas se dedicaban a su producción, y a pesar de que está disminuyendo este número por la contracción agrícola, la proporción del caso del arroz sobresale todavía. De igual forma que en la proporción de la superficie de terreno cultivable, el porcentaje de cultivo de arroz es mayor y las unidades familiares agrícolas que se dedican a su cultivo son superiores (tabla 5).

\section{Tabla 5}

Porcentaje de familias rurales que se dedican al cultivo del arroz (unidad: 1,000, familia \%)

\begin{tabular}{lccc}
\hline Año & $\begin{array}{c}\text { Total de unidad de familias } \\
\text { agrícolas }\end{array}$ & $\begin{array}{c}\text { Cantidad de unidades } \\
\text { familiares que cultivan arroz }\end{array}$ & $\%$ \\
\hline 1990 & 1767 & 1525 & 86.3 \\
1995 & 1501 & 1205 & 80.2 \\
2000 & 1383 & 1078 & 77.9 \\
2004 & 1264 & 945 & 74.7 \\
\hline
\end{tabular}

Fuente: elaboración propia con datos de Estadística Anual Agropecuaria 2005.

Como se observa en la tabla 5, en 2004, de las 1'264,000 unidades familiares agrícolas, 945,000 cultivaban arroz (74.7\% del total); si lo comparamos con el año 1990, la cantidad de unidades familiares disminuyó. En la década de 1990, muchos campesinos sufrieron por la política de la apertura agrícola y una parte de ellos abandonaron sus actividades, razón por la cual el arroz de Corea no pudo competir con otros países, debido al costo de su producción. Por ejemplo, en EE.UU. es cinco veces más barato que en Corea. Después de la Ronda Uruguay, el gobierno de Corea tuvo que liberar la actividad agrícola, iniciando con la carne, las frutas y los granos básicos... menos el arroz. Así, en la década de 1990 este producto todavía no estaba liberado, pero se enfrentaba una gran depresión internacional. Ante esta situación, algunos campesinos cambiaron su actividad económica; sin embargo, en la actualidad el arroz ocupa un mayor porcentaje de la unidad familiar total.

El porcentaje de arroz en el consumo calórico total de los coreanos

En Corea la comida básica es, por supuesto, el arroz: ocupa el primer lugar no sólo entre los cereales, sino también entre todo tipo de alimentos. Aunque han existido cambios de acuerdo a la situación de cada época, sigue siendo el arroz la primera fuente de calorías de los coreanos (tabla 6). 


\section{Tabla 6}

Contribución de calorías de los principales alimentos en la dieta de los coreanos (unidad: Kcal, \%)

\begin{tabular}{|c|c|c|c|c|c|c|c|c|c|}
\hline Año & Total & Arroz & Trigo & Cebada & Papa & Soya & Vegetales & Carne & Mariscos \\
\hline 1970 & 2370 & $\begin{array}{l}1215 \\
(51.3)\end{array}$ & $\begin{array}{c}243 \\
(10.2)\end{array}$ & $\begin{array}{c}335 \\
(14.1)\end{array}$ & $\begin{array}{c}180 \\
(7.6)\end{array}$ & $\begin{array}{c}85 \\
(3.6)\end{array}$ & $\begin{array}{c}53 \\
(2.2)\end{array}$ & $\begin{array}{c}49 \\
(2.0)\end{array}$ & $\begin{array}{c}40 \\
(1.7)\end{array}$ \\
\hline 1975 & 2390 & $\begin{array}{c}1116 \\
(46.7)\end{array}$ & $\begin{array}{c}292 \\
(12.2)\end{array}$ & $\begin{array}{c}361 \\
(15.1)\end{array}$ & $\begin{array}{l}129 \\
(5.4)\end{array}$ & $\begin{array}{c}90 \\
(3.8)\end{array}$ & $\begin{array}{c}57 \\
(2.4)\end{array}$ & $\begin{array}{c}50 \\
(2.0)\end{array}$ & $\begin{array}{c}66 \\
(2.8)\end{array}$ \\
\hline 1980 & 2485 & $\begin{array}{c}1234 \\
(49.7)\end{array}$ & $\begin{array}{c}284 \\
(11.4)\end{array}$ & $\begin{array}{l}129 \\
(5.2)\end{array}$ & $\begin{array}{c}65 \\
(2.6)\end{array}$ & $\begin{array}{c}103 \\
(4.1)\end{array}$ & $\begin{array}{l}119 \\
(4.8)\end{array}$ & $\begin{array}{c}91 \\
(3.7)\end{array}$ & $\begin{array}{c}61 \\
(2.5)\end{array}$ \\
\hline 1985 & 2687 & $\begin{array}{l}1245 \\
(46.3)\end{array}$ & $\begin{array}{c}307 \\
(11.4)\end{array}$ & $\begin{array}{c}85 \\
(3.2)\end{array}$ & $\begin{array}{c}30 \\
(1.1)\end{array}$ & $\begin{array}{l}115 \\
(4.3)\end{array}$ & $\begin{array}{c}97 \\
(3.6)\end{array}$ & $\begin{array}{l}100 \\
(3.7)\end{array}$ & $\begin{array}{c}92 \\
(3.4)\end{array}$ \\
\hline 1990 & 2853 & $\begin{array}{c}1175 \\
(41.2)\end{array}$ & $\begin{array}{c}285 \\
(10.0)\end{array}$ & $\begin{array}{c}22 \\
(0.8)\end{array}$ & $\begin{array}{c}27 \\
(0.9)\end{array}$ & $\begin{array}{c}112 \\
(3.9)\end{array}$ & $\begin{array}{c}116 \\
(4.0)\end{array}$ & $\begin{array}{l}143 \\
(5.0)\end{array}$ & $\begin{array}{c}92 \\
(3.2)\end{array}$ \\
\hline 1995 & 2959 & $\begin{array}{c}1054 \\
(35.6)\end{array}$ & $\begin{array}{c}343 \\
(11.6)\end{array}$ & $\begin{array}{c}18 \\
(0.6)\end{array}$ & $\begin{array}{c}29 \\
(1.0)\end{array}$ & $\begin{array}{l}117 \\
(3.9)\end{array}$ & $\begin{array}{l}127 \\
(4.3)\end{array}$ & $\begin{array}{l}189 \\
(6.4)\end{array}$ & $\begin{array}{c}99 \\
(3.3)\end{array}$ \\
\hline 2000 & 3010 & $\begin{array}{c}997 \\
(33.1)\end{array}$ & $\begin{array}{c}363 \\
(12.0)\end{array}$ & $\begin{array}{c}17 \\
(0.5)\end{array}$ & $\begin{array}{c}29 \\
(1.0)\end{array}$ & $\begin{array}{l}116 \\
(3.8)\end{array}$ & $\begin{array}{c}126 \\
(4.1)\end{array}$ & $\begin{array}{c}207 \\
(6.9)\end{array}$ & $\begin{array}{c}87 \\
(2.9)\end{array}$ \\
\hline 2003 & 2983 & $\begin{array}{c}893 \\
(29.9)\end{array}$ & $\begin{array}{c}329 \\
(11.0)\end{array}$ & $\begin{array}{c}11 \\
(0.4)\end{array}$ & $\begin{array}{c}30 \\
(1.0)\end{array}$ & $\begin{array}{l}110 \\
(3.7)\end{array}$ & $\begin{array}{l}118 \\
(3.9)\end{array}$ & $\begin{array}{c}211 \\
(7.0)\end{array}$ & $\begin{array}{l}109 \\
(3.7)\end{array}$ \\
\hline
\end{tabular}

Fuente: Elaboración propia con datos de Estadística Anual Agropecuaria 2005.

En la tabla 6 se constata que, durante los últimos treinta años, el arroz ha ocupado el primer lugar entre todos los productos que componen el consumo de alimentos, medido en calorías, de los coreanos; aunque su porcentaje disminuyó de $51.3 \%$ hasta $29.9 \%$ durante este periodo, continua siendo el alimento principal como fuente de calorías. En este tiempo, la suma de calorías adquiridas a través de la carne, mariscos y vegetales aumentó, mientras que en el caso de la cebada, papa y camote disminuyó. De esta manera, el desarrollo económico en el último cuarto del siglo xx indujo el cambio en los hábitos alimenticios, y el mejoramiento del nivel de vida de los coreanos les permitió consumir más carne, mariscos y vegetales.

Aparte de los productos que se muestran en la tabla 6, otros granos ocuparon 10.9\%, el azúcar 7.4\%, y los aceites y grasas 13.7\% en 2003.

Así pues, desde la década de 1980 la cantidad de consumo de arroz disminuyó paulatinamente, mientras que la cantidad del consumo de carne, productos lácteos, verduras y frutas se incrementó. Esto significa que la dieta alimenticia de los coreanos cambió, dejando a un lado los alimentos a base de 
cereales, por los elaborados a base de carnes y vegetales. Sin embargo, para el año 2004 la comida básica de los coreanos seguía siendo el arroz (tabla 7).

Tabla 7

El consumo anual de los alimentos per cápita en Corea (unidad: kg)

\begin{tabular}{lrrcccccc}
\hline Año & Arroz & Trigo & Carne de res & Carne de puerco & Pollo & Leche & Verdura & Fruta \\
\hline 1980 & 132.4 & 29.4 & 2.6 & 6.3 & 2.4 & 10.8 & 120.3 & 22.3 \\
1985 & 128.1 & 32.1 & 2.9 & 8.4 & 3.1 & 23.8 & 98.6 & 36.0 \\
1990 & 119.6 & 29.8 & 4.1 & 11.8 & 4.0 & 42.8 & 132.6 & 41.8 \\
1995 & 106.5 & 33.4 & 6.7 & 14.8 & 5.9 & 47.8 & 160.6 & 55.4 \\
2000 & 93.6 & 35.8 & 8.5 & 16.1 & 6.9 & 59.2 & 165.9 & 58.4 \\
2002 & 87.0 & 34.6 & 8.5 & 17.0 & 8.0 & 64.2 & 144.6 & 58.8 \\
2004 & 82.0 & 34.1 & 6.8 & 17.9 & 6.6 & 63.7 & & 58.8 \\
\hline
\end{tabular}

Fuente: elaboración propia con datos en la Estadística Anual Agropecuaria 2005.

El análisis de la tabla 7 nos permite observar que desde la década de 1980 el consumo del arroz disminuyó drásticamente. En esta época los coreanos consumían $132.4 \mathrm{~kg}$ de arroz, pero en 2004, los coreanos sólo consumieron $82 \mathrm{~kg}$ por año. En el caso del trigo, la cantidad de consumo anual aumentó durante en el mismo periodo. De igual manera pasó con todo tipo de carnes. Además, el consumo de leche y frutas fue mayor que el de otros alimentos. Todo esto demuestra el incremento en el nivel de vida de los coreanos, quienes ahora consumen más carne, leche y frutas. No obstante lo anterior, en comparación con otros países la cantidad de consumo de estos productos es muy baja, ${ }^{4}$ y el arroz sigue siendo la comida básica en Corea.

La proporción de arroz entre el valor total agrícola

El porcentaje del arroz en el PIB agrícola es muy pequeño, comparado con el porcentaje de la superficie cultivada, así como con el porcentaje de la unidad familiar agrícola dedicada a su cultivo. En el año 2004, 81\% del terreno cultivable se utilizó para la producción de arroz y $74.7 \%$ de la unidad familiar

4. En el caso de carne de res, en 2004, en EE.UU. el consumo anual per capita fue de $42.8 \mathrm{~kg}$ y en Australia de $38.3 \mathrm{~kg}$. 
agrícola se dedicó a su cultivo. Sin embargo, en la producción de valor agrícola (PIB agrícola), el arroz ocupa 27.6\% (tabla 8).

Tabla 8

La proporción del arroz entre PIB agrícola

\begin{tabular}{cccc}
\hline Año & $\%$ & Año & $\%$ \\
\hline 1990 & 39.9 & 2000 & 33.0 \\
1995 & 26.1 & 2001 & 33.0 \\
1996 & 30.6 & 2002 & 29.7 \\
1998 & 31.0 & 2003 & 27.8 \\
1999 & 31.5 & 2004 & 27.6 \\
\hline
\end{tabular}

Fuente: elaboración propia con datos en la Estadística Anual Agropecuaria 2005.

De acuerdo a la tabla 8, de 1990 a 2004 la proporción de valor del arroz entre la producción total agrícola disminuyó en 12.3 puntos, siendo la causa de esta situación la baja producción y su valor económico. En la tabla 9 se observa que en las últimas décadas la producción de arroz disminuyó, mientras que la del ganado y los vegetales se incrementó.

En esta época, el precio del arroz no subió tanto como el del ganado y las hortalizas, situación que se refleja en el cambio del porcentaje del ingreso por arroz entre el PIB agrícola.

Tabla 9

Ingreso agrícola por cada producto agrícola (unidad: \%)

\begin{tabular}{ccccc}
\hline Año & Arroz & Cebada & Vegetal y fruta & Ganado \\
\hline 1980 & 48.7 & 5.1 & 25.7 & 12.1 \\
1985 & 48.0 & 2.3 & 28.0 & 17.0 \\
1990 & 48.2 & 1.9 & 28.1 & 17.5 \\
1995 & 34.0 & 1.2 & 35.8 & 24.9 \\
2000 & 39.8 & 0.9 & 41.2 & 13.1 \\
2002 & 37.4 & 1.2 & 43.1 & 12.8 \\
\hline
\end{tabular}

Fuente: Seong, 2004.

En el ingreso total agrícola, el arroz ocupó 37.4\% en 2002 y hasta 1990 ocupaba un mayor porcentaje en el ingreso total agrícola. Sin embargo, desde 
hace 15 años, los vegetales y frutas sobresalen en el ingreso total agrícola, rebasando el porcentaje del arroz. Para 2004, el ingreso que proviene de ellos ocupó $43 \%$, aunque el porcentaje de unidades familiares agrícolas (UFA) dedicadas a estos cultivos nada más alcanzó $27.6 \%$. Además, las unidades agrícolas dedicadas a la ganadería representaba 5.2\% de las UFA, pero su ingreso ocupó $12.8 \%$. Esta situación significa que hoy el arroz no tiene tanta demanda entre los productores; sin embargo los campesinos coreanos no pueden dejar su cultivo, por razones culturales e históricas.

\section{Autosuficiencia del arroz}

En la década de 1970, la tasa de autosuficiencia alimentaria fue de más de $80 \%$, pero en 2004 , la tasa de autosuficiencia quedó en $26.8 \%$. En el caso de los cereales, el porcentaje fue el más bajo del promedio. En 2004 la cebada ocupó $54.1 \%$, pero el trigo y el maíz ocuparon $0.4 \%$ y $0.8 \%$, respectivamente. $\mathrm{Si}$ consideramos la tasa de autosuficiencia de otros países, estos niveles son muy bajos. ${ }^{5}$

En el caso del arroz la tasa de autosuficiencia es todavía muy alta, casi llega a $100 \%$ (tabla 10).

Tabla 10

Porcentaje de autosuficiencia de los cereales (unidad: \%)

\begin{tabular}{lcrrrrrr}
\hline Año & Total & Arroz & Cebada & \multicolumn{1}{c}{ Trigo } & \multicolumn{1}{c}{ Maíz } & \multicolumn{1}{c}{ Soya } & Papas \\
\hline 1965 & - & 100.7 & 106.0 & 27.0 & 36.1 & 100.0 & 100.0 \\
1970 & 80.5 & 93.1 & 106.3 & 15.4 & 18.9 & 86.1 & 100.0 \\
1975 & 73.1 & 94.6 & 92.0 & 5.7 & 8.3 & 85.8 & 100.0 \\
1980 & 56.0 & 95.1 & 57.6 & 4.8 & 5.9 & 35.1 & 100.0 \\
1985 & 48.4 & 103.3 & 63.7 & 0.4 & 4.1 & 22.5 & 100.0 \\
1990 & 43.1 & 108.3 & 97.4 & 0.05 & 1.9 & 20.1 & 95.6 \\
1995 & 29.1 & 91.4 & 67.0 & 0.3 & 1.1 & 9.9 & 98.4 \\
2000 & 29.7 & 102.9 & 46.9 & 0.1 & 0.9 & 6.4 & 99.3 \\
2004 & 26.8 & 96.5 & 54.1 & 0.4 & 0.8 & 7.1 & 97.1 \\
\hline
\end{tabular}

Fuente: elaboración propia con datos de Byun (1996) y la Estadística Anual Agropecuaria 2005.

5. En el año 2002, EE.UU. tenía una tasa de autosuficiencia de cereal de $118.9 \%$; en tanto que Gran Bretaña, Canadá, Dinamarca, Francia y Alemania, 109.0\%, 119.7\%, 106.7\%, 186.8\%, y $111.4 \%$, respectivamente. 
Se puede observar en la tabla 10 que la tasa de autosuficiencia del arroz se mantuvo durante los últimos cuarenta años en más de $90 \%$, mientras que la de otros cereales disminuía drásticamente: desde $80.5 \%$ hasta $26.8 \%$ en el mismo periodo. El caso de la soya puede ser un buen ejemplo, ya que en el año 1965 la tasa de autosuficiente de la soya fue de 100\%, pero en el año 2004 bajó hasta $7.1 \%$. Hoy en día los cereales como cebada, trigo, maíz y soya integran la comida básica, más su producción no es suficiente para satisfacer el autoconsumo, por lo que para completarlo se realizan importaciones. En realidad, en 2004 la tasa de autosuficiencia del arroz alcanzó 96.5\%, pero la autosuficiencia en cereales fue solamente de $26.8 \%$.

\section{La estructura y característica de la sociedad agrícola coreana: disminución y envejecimiento de la población agrícola}

En la sección anterior analizamos la estructura y características de la propiedad y producción agrícola. En ésta se analizan la estructura y características de la sociedad agropecuaria, puesto que el perfil demográfico puede ser el elemento básico en la sociedad estudiada. Desde tal perspectiva, la presente sección analiza los fenómenos de disminución y envejecimiento de la población agrícola.

Disminución de población agrícola

En la segunda mitad del siglo xx, la población agrícola disminuyó abruptamente debido a la industrialización, debido que gran parte emigró a la zona urbana (tabla 11).

\section{Tabla 11}

Éxodo rural a las ciudades en Corea, 1960-1990

(unidad: miles de campesinos)

\begin{tabular}{cc}
\hline Periodo & Número \\
\hline $1961-1965$ & 434 \\
$1966-1970$ & 1506 \\
$1971-1975$ & 1196 \\
$1976-1980$ & 1843 \\
$1981-1985$ & 1535 \\
$1986-1990$ & 1594 \\
\hline
\end{tabular}

Fuente: elaboración propia, con datos de Seol (2002). 
Como se ve en la tabla 11, en la segunda mitad del siglo xx más de 10 millones de personas que vivían en las zonas rurales salieron de su lugar agrícola. En consecuencia, la proporción de la población rural entre la total disminuyó de $50.7 \%$ hasta $12 \%$ entre 1971 y 2004 . Este fenómeno se reflejó en la reducción de la unidad de producción agrícola familiar. Un ejemplo es el año de 1961, cuando la proporción de la unidad de producción agrícola familiar ocupaba 53.6\%, de las unidades familiares en Corea; pero ya en 2004, la unidad familiar agrícola ocupó sólo $8 \%$ del total. Así pues, el número de la unidad familiar agrícola disminuyó en la segunda mitad de siglo xx, mientras que la unidad familiar total aumentaba (tabla 12).

Tabla 12

Cambio del número de la unidad de producción agrícola familiar (unidad: 1,000 unidad familiar)

\begin{tabular}{cccc}
\hline Año & Unidad familiar total & Unidad producción agrícola familiar & $\%$ \\
\hline 1961 & - & 2327 & 53.6 \\
1965 & - & 2507 & 50.3 \\
1970 & 5857 & 2488 & 42.9 \\
1975 & 6754 & 2379 & 35.2 \\
1980 & 7969 & 2155 & 27.1 \\
1985 & 9571 & 1926 & 20.1 \\
1990 & 11355 & 1767 & 15.6 \\
1995 & 12958 & 1501 & 11.6 \\
2000 & 14312 & 1383 & 9.7 \\
2004 & 15539 & 1240 & 8.0 \\
\hline
\end{tabular}

Fuente: elaboración propia, con datos de Byun (1996) y de la Estadística Anual Agropecuaria 2005.

La reducción de la cantidad de la población agrícola se refleja en la disminución del porcentaje de la producción agrícola entre producción total y la del empleo agrícola entre empleo total. En el PIB total, la producción agrícola bajó de $36.8 \%$ en 1960 , hasta $4.7 \%$ en 2000 . Así mismo, el cambio en el porcentaje del empleo agrícola disminuyó más que el cambio en la estructura económica de Corea. Entre 1963 y 2004, la proporción del empleo agrícola bajó de 63.1\% hasta $8.1 \%$ (tablas 13 y 14 ). 
Tabla 13

Cambio de la estructura económica en Corea, 1960-2000

\begin{tabular}{cccc}
\hline Año & Sector primario & Sector secundario & Sector terciario \\
\hline 1960 & 36.8 & 3.8 & 49.4 \\
1970 & 27.1 & 21.2 & 51.7 \\
1980 & 14.8 & 28.2 & 54 \\
1990 & 8.5 & 28.8 & 62.7 \\
2000 & 4.7 & 31.3 & 64 \\
\hline
\end{tabular}

Fuente: Kang (2005: 79).

Tabla 14

Cambio de la estructura del empleo, 1963 -2004 (unidad: \%)

\begin{tabular}{lccc}
\hline Año & Sector primario & Sector secundario & Sector terciario \\
\hline 1963 & 63.1 & 8.7 & 28.2 \\
1965 & 58.6 & 10.4 & 31.0 \\
1970 & 50.4 & 14.3 & 35.3 \\
1975 & 45.7 & 19.1 & 35.2 \\
1980 & 34.0 & 22.5 & 43.5 \\
1985 & 24.9 & 24.4 & 50.6 \\
1990 & 17.9 & 27.6 & 54.5 \\
1995 & 11.8 & 23.7 & 64.5 \\
2000 & 10.6 & 20.4 & 69.0 \\
2004 & 8.1 & 19.1 & 72.8 \\
\hline
\end{tabular}

Fuente: elaboración propia, con datos de Byun (1996) y de la Estadística Anual Agropecuaria 2005.

La velocidad del cambio en la proporción de producto y empleo fue grande en Corea, y ha sido una característica de la agricultura coreana. Si se compara con otros países, esta velocidad se vuelve más notoria (tabla 15). 
Tabla 15

Cambio de la proporción del PIB agrícola y la duración del tránsito de $40 \%$ a $7 \%$

\begin{tabular}{lccc}
\hline País & Año con $40 \%$ & Año con $7 \%$ & Duración de años \\
\hline Gran Bretaña & 1788 & 1901 & 113 \\
Holanda & Cerca de 1800 & 1965 & 165 \\
Alemania & 1854 & 1950 & 96 \\
EE.UU. & 1866 & 1958 & 92 \\
Dinamarca & 1850 & 1969 & 119 \\
Francia & 1878 & 1972 & 94 \\
Japón & 1896 & 1969 & 73 \\
Corea & 1965 & 1991 & 26 \\
\hline
\end{tabular}

Fuente: Lee y Lim (2004).

La tabla 15 muestra lo que tardó cada país para pasar de 40\% hasta 7\% del PIB agrícola entre PIB total. Como se observa, entre los países europeos, Gran Bretaña llegó primero a $40 \%$ en 1788 , y las otras naciones lo alcanzaron en la segunda mitad del siglo xIX. Si calculamos el tiempo en que cada país tardó para llegar a 7\%, tenemos que Holanda demoró más años que los demás, con 165. Por su parte, Gran Bretaña y Dinamarca duraron más de 100 años; en el caso de EE.UU. llevó 92 años y en Japón 73. Pero si vemos el caso de Corea, en el año 1965 llegó a 40\% y exactamente después de 26 años, en 1991, alcanzó $7 \%$. Si comparamos a Corea con los demás países, el tiempo que tardó fue más corto, como se puede observar en el gráfico 2.

\section{Gráfico 2}

Cambio de la proporción PIB agrícola

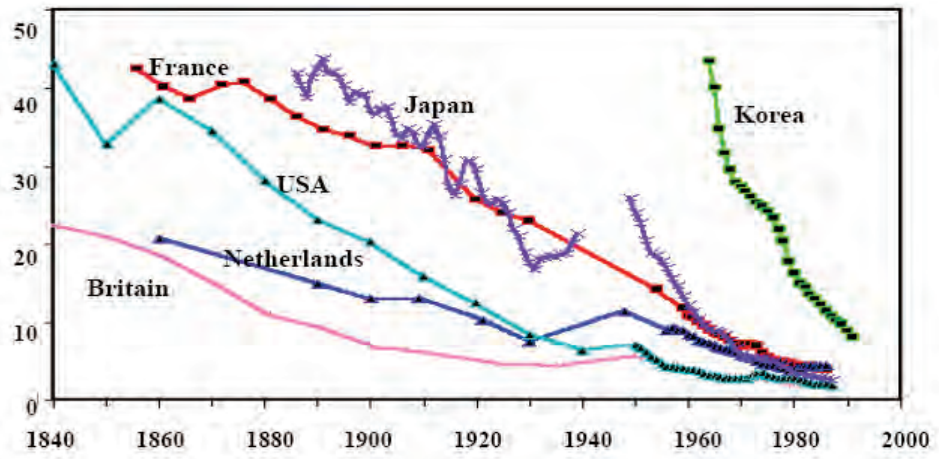

Fuente: Lee y Lim (2004). 
Con la modificación del porcentaje de producción agrícola, podemos ver el cambio del empleo agrícola. Como se ve en la tabla 14, en Corea el cambio del empleo agrícola se desarrolló muy rápidamente. Para comparar esta tendencia con otros países, observemos la tabla 16, realizada por Lee y Lim (2004), donde se muestra lo que tardó cada país en transitar de $40 \%$ hasta $16 \%$.

\section{Tabla 16}

Cambio de la proporción del empleo agrícola y la duración del tránsito de $40 \%$ a $16 \%$

\begin{tabular}{lccc}
\hline País & Año con $40 \%$ & Año con $16 \%$ & Duración de años \\
\hline Gran Bretaña & Antes de 1800 & 1868 & Más de 70 \\
Holanda & 1855 & 1950 & 95 \\
Alemania & 1897 & 1957 & 60 \\
EE.UU. & 1900 & 1942 & 42 \\
Dinamarca & 1920 & 1962 & 42 \\
Francia & 1921 & 1965 & 44 \\
Japón & Cerca de 1940 & 1971 & Alrededor de 31 \\
Corea & 1977 & 1991 & 14 \\
\hline
\end{tabular}

Fuente: Lee y Lim (2004).

Al igual que en el caso de la participación de la producción agrícola, la velocidad del cambio del empleo agrícola fue más rápida en Corea que en otros países. En Holanda, el tránsito de 40\% hasta 16\% tardó 95 años. Gran Bretaña y Alemania duraron 70 y 60 años respectivamente, mientras que EE.UU., Dinamarca y Francia duraron más de 40 años. En el caso de Japón, el desarrollo fue más rápido que los países europeos, duró 31 años; Corea solamente tardó 14 años. Estas tendencias se muestran en el gráfico 3.

En conclusión, en Corea la velocidad del cambio de la proporción del empleo agrícola fue más rápido que en los países europeos, EE.UU. y los países asiáticos. Aunque la industrialización y la urbanización se iniciaron en este país más tarde que en los demás, causa por la que la agricultura se mantenía muy fuerte hasta la segunda mitad del siglo xx, la velocidad del cambió fue mayor.

\section{Envejecimiento}

Con la disminución de la población rural, el envejecimiento podría ser uno de los problemas de la sociedad agrícola coreana. Hasta el año 1960, la población 


\section{Gráfico 3}

Cambio de la proporción del empleo agrícola

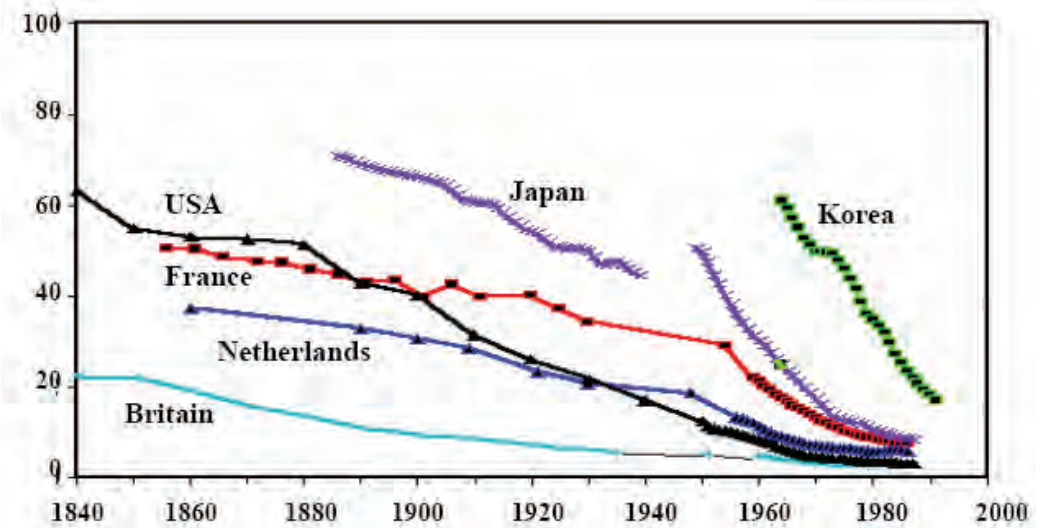

Fuente: Lee y Lim (2004).

rural era de 18 millones, mientras que la población urbana sólo alcanzaba siete millones. Pero después de cuarenta años, en 2000 la población rural disminuyó hasta 9.3 millones, mientras que la población urbana alcanzó los 36.6 millones. Durante estos años la población rural disminuyó dos veces, mientras que la población urbana aumentó cinco.

Concretamente, por causa de la industrialización, desde la década de 1970 muchos pobladores rurales emigraron a la zona urbana; especialmente los jóvenes, que fueron absorbidos por los sectores secundarios y terciarios; sucedió lo contrario con los adultos, quienes se quedaron en la zona rural atendiendo la agricultura. A causa del éxodo de los jóvenes, la reproducción poblacional disminuyó en la zona rural y el perfil demográfico envejeció, fenómeno que se muestra en el gráfico 4 .

En este gráfico se muestra la estructura de edad en la zona rural y urbana entre 1960 y 2000. Así en 1960, 6.7\% de la población rural ocupaba el rango de edad de más de 60 años, pero en 2000, más de $22.3 \%$ de la población rural era mayor de 60 años. Al contrario, el rango de edad de los jóvenes (10-19, 20-29, 30-39, y 40-49) se incrementó en la zona urbana. Este fenómeno del envejecimiento de la población rural se analiza detalladamente con los datos de la edad de los dueños del terreno agrícola (tabla 17). 


\section{Grafico 4}

El perfil de la demografía en la zona urbana y rural entre 1960 y 2000
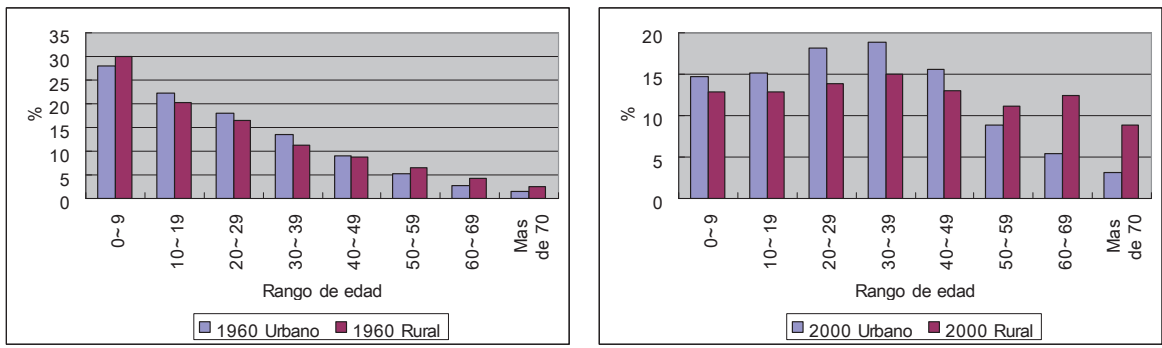

Fuente: elaboración propia, con datos de la Estadística Anual de Corea.

Tabla 17

La distribución por rango de edad, de los jefes de familia de la unidad agrícola (unidad: 1,000, \%)

\begin{tabular}{lcccccc}
\hline Rango de edad & Total & Menos 40 & $40-49$ & $50-59$ & $60-69$ & Más 70 \\
\hline Número & 1240 & 38 & 182 & 286 & 449 & 285 \\
$\%$ & 100.0 & 3.0 & 14.7 & 23.1 & 36.2 & 23.0 \\
\hline
\end{tabular}

Fuente: cuadro de elaboración propia con datos de la Estadística Anual Agropecuaria 2005.

Como se observa en esta tabla, los dueños de unidades de producción agrícola de menos de 40 años ocupan sólo 3\%; al contrario del porcentaje de edad de más de 60 años, que alcanzó 59.2\%. Con base en esta situación, podemos decir que el fenómeno de envejecimiento es muy fuerte en la sociedad agrícola de Corea.

El fenómeno del envejecimiento sobresale en el caso del cultivo de arroz, de entre todos los tipos de productos agrícolas (tabla 18).

Según datos de la tabla 18, la suma de todas la unidades familiares agrícolas es de 1'204,000. Entre ellas, 59.2\% son atendidas por el dueño que tiene más de 60 años, pero en los casos de la floricultura y la ganadería nada más $28.4 \%$ y $38.7 \%$ de los dueños tienen más de 60 años, por lo que podemos decir que en estos casos los dueños son más jóvenes que el promedio. En cuanto a la producción de frutas y vegetales, las edades de los dueños de las unidades de producción son mayores que en los casos de los floricultores y ganaderos; 
Tabla 18

Perfil, por rango de edad, de los dueños de unidades de producción en varios tipos de productos agrícolas, en 2004 (unidad: 1,000 unidad de producción, \%)

\begin{tabular}{lccccccc}
\hline & Total & Arroz & Fruta & Vegetal & Flores & Ganado & Otro \\
\hline Unidad total (\%) & 1204 & 640 & 136 & 262 & 11 & 83 & 109 \\
& $(100.0)$ & $(100.0)$ & $(100.0)$ & $(100.0)$ & $(100.0)$ & $(100.0)$ & $(100.0)$ \\
Menos de 59 & 506 & 230 & 62 & 113 & 8 & 51 & 43 \\
años (\%)* & $(40.8)$ & $(36.0)$ & $(45.4)$ & $(43.3)$ & $(71.6)$ & $(61.3)$ & $(40.5)$ \\
Más de 60 años & 734 & 409 & 74 & 148 & 3 & 32 & 68 \\
(\%)** $^{(59.2)}$ & $(64.0)$ & $(54.6)$ & $(56.7)$ & $(28.4)$ & $(38.7)$ & $(59.5)$ \\
\hline
\end{tabular}

* Número de la unidad de producción familiar que tiene dueño con edad menor a 59 años.

**Número de la unidad de producción familiar que tiene dueño con edad mayor a 60 años. Fuente: KREI (2006).

sin embargo, están por debajo del promedio. Pero con el arroz, la proporción del rango de edad de más de 60 años ocupa $64 \%$, siendo mayor que el promedio. De esta manera podemos decir que entre todo los tipos de producciones agrícolas, el fenómeno del envejecimiento es mayor en el caso del arroz.

Así pues, la causa de que los jóvenes prefieren producir frutas y criar ganado, en lugar de cultivar arroz, es la diferencia del ingreso en cada tipo de actividad.

Tabla 19

Contribución porcentual del ingreso agrícola total por productos (unidad: \%)

\begin{tabular}{lrrrrrr}
\hline Año & Grano (arroz) & Vegetal & \multicolumn{1}{l}{ Fruta } & Otro & Ganado & Total \\
\hline \multicolumn{2}{l}{ Proporción del valor de producción agrícola } \\
\hline 1990 & 41.2 & 18.2 & 8.2 & 10.1 & 22.3 & 100.0 \\
1995 & 32.8 & 23.6 & 10.4 & 9.6 & 23.5 & 100.0 \\
2000 & 35.8 & 21.6 & 8.2 & 9.1 & 25.3 & 100.0 \\
2003 & 31.4 & 22.0 & 7.9 & 10.0 & 28.7 & 100.0 \\
\hline Tasa de crecimiento & & & & & & \\
\hline $90 \sim 95$ & -4.41 & 5.36 & 4.96 & -0.82 & 1.09 & 0.04 \\
$95 \sim 00$ & 2.67 & -0.85 & -3.79 & -0.30 & 2.43 & 0.91 \\
$00 \sim 03$ & -5.54 & -0.76 & -2.71 & 1.83 & 2.89 & -1.35 \\
$90 \sim 03$ & -2.02 & 1.51 & -0.23 & -0.01 & 2.02 & 0.05 \\
\hline
\end{tabular}

Fuente: KREI (2006). 
En el año 2000 la proporción de la producción agrícola de arroz alcanzó $33 \%$, mientras $73.7 \%$ de la unidad familiar agrícola se dedicaba a su producción y en más de $80 \%$ del terreno cultivable se sembraba este producto. La actividad ganadera alcanzó $25.3 \%$ de la producción total agrícola, mientras que $5.2 \%$ de la unidad familiar agrícola se dedicaba al ganado (Kim, 2002), lo que demuestra que el ingreso agrícola con el ganado es más productivo que con el arroz, justificación para que los jóvenes buscaran dedicarse a la ganadería en lugar del cultivo de arroz, razón por la cual el promedio de edad de los dueños de los campos ganaderos es menor. Sin embargo, cabe destacar que en la ganadería la fuerza de trabajo es mayor, así como la cantidad de inversión. Por lo tanto, los dueños que tienen edad avanzada no pueden dedicarse al ganado.

\section{Conclusión}

En este artículo se analizaron la estructura y características de la agricultura de Corea en la segunda mitad del siglo xx, concretamente en el campo de la propiedad, la producción y la sociedad agrícola coreana. Algunos de los resultados obtenidos son:

Primero, la estructura coreana de la propiedad del terreno agrícola se caracteriza por su pequeño tamaño; así, en el año 2005 el promedio de la propiedad del terreno quedó en 1.3 ha. Comparado con otros países, por ejemplo EE.UU., Canadá, y Australia, podemos decir que el tamaño es demasiado pequeño, siendo esto un factor importante para explicar la situación agrícola de Corea.

Segundo, con relación a la producción agrícola, podemos decir que el arroz es el pivote principal en la agricultura de Corea. De la tierra cultivable, $81 \%$ se dedica al arroz y $74.7 \%$ de las unidades familiares agrícolas se dedican a su producción. Además, entre todas las calorías que consumen los coreanos, el arroz es la principal fuente; con relación a la tasa de autosuficiencia alimenticia, éste alcanza casi $100 \%$, mientras que el promedio es de $26 \%$. Por lo tanto, podemos decir que en la producción agrícola de Corea el arroz funciona como el principal pivote.

Tercera, en la segunda mitad del siglo xx, debido a la industrialización y urbanización, la población rural disminuyó considerablemente, por lo que a finales del siglo xx se inició el envejecimiento en la población rural. Este fenómeno fue mayor en la producción del arroz en comparación con la actividad ganadera y de producción frutal.

En conclusión, en este artículo se confirmó que la agricultura de Corea tiene características muy diferenciadas en comparación con otros países en 
relación a la propiedad y producción. Podemos afirmar que la agricultura coreana se desarrolló con base en una pequeña escala, donde la mayor parte se dedicaba a la producción del arroz, mientras que a la fecha, la sociedad agrícola está sufriendo la disminución y envejecimiento de la población.

A pesar de que la agricultura ha fungido como el principal factor económico para el desarrollo de Corea, en la actualidad los jóvenes prefieren el trabajo industrial al del campo. my

\section{Referencias bibliográficas}

Byun, Hyungyoon, Economía coreana (한국경 제론), Editorial Yupoong, Corea, 1996.

Chang SAng Hwan, "La reforma agrícola y el desarrollo del capitalismo coreano" (농지개혁과 한국자본주의 발전), Investigación de Desarrollo Económico, vol. 6, núm. 1, Corea, 2000.

KANG, Insu (ed.), Comprensión de la economía contemporánea coreana (한국경 제의 이해), Editorial Kyobo, Corea, 2005.

KIM, Byungteak, Política agrícola en Corea (한국의 농업 정책), Editorial Hanul, Corea, 2002.

KIM, Jeongho, El cambio de la estructura agricultura de arroz: tendencia y perspectiva (쌀 농업구조변화:동향과 전망), KREI, Corea, 2004.

KIM Dae re, Compresión de la economía contemporánea coreana (현 대 한국경 제의 이해), Editorial Shingi, Corea, 2005.

Korea RuRAl ECONOMIC Institute (KREI), Perspectiva de la agricultura en Corea 2006 (농업 전망 2006), Korea Rural Economic Institute, Corea, 2006.

LEE, Jeonghwan y Songsoo Lim, El camino futuro para la agricultura coreana (The road ahead for Korean agriculture: soft landing on a new plateau in the WTO), Korea Rural Economic Institute, Corea, 2004.

LEE, Jeonghwan, El principio y el término del cambio de la estructura agrícola ( 농업구조 전환 그 시작과 끝), KREI, Corea, 1998.

Ministerio DE AGRICULTURA, Estadística anual agrícola (농업 통계), Ministerio de Agricultura, Corea, 2005.

SEOL, Donghun, Los trabajadores extranjeros en la sociedad de Corea 「외국 인노동자와「한국사회「, Editorial de la Universidad Nacional de Seúl, Corea, 2002.

SEONG, Jinkeun (ed.), Vamos cambiar la Política agrícola en Corea (한국의 농 업정책, 틀을 바꾸자), Samsung Economic Institute, Corea, 2004. 